\title{
Designing of New Data Synchronization System
}

\author{
Jinbin Yang, Jianping Zheng, Yanlei Shang, Junliang Chen \\ The State Key Lab of Networking and Switching, Beijing University of Posts and Telecommunications, Beijing, China \\ Email: jackieyang1987@163.com, zhengjianping@chinamobile.com, shangyl@bupt.edu.cn, chjl@bupt.edu.cn
}

Received 2012

\begin{abstract}
As the rapid development of Wireless Communications and the popularity of the Intelligent Terminal, data synchronization has been a social focus, meanwhile, user terminal devices are increasingly diversified, traditional synchronization technology based C/S mode has such deficiencies as insufficient amount of transmitting data and bad Real-time efficiency. It has become increasingly unable to meet the needs of future development. In this paper, we proposed and designed a new method and system by separating control with transmission to synchronize data to ensure Real-time data and improve efficiency.
\end{abstract}

Keywords: Mobile Internet; Wireless Communications; Data Synchronization; Control; Transmission

\section{Introduction}

With the development of mobile communication technology and mobile terminal intelligence, mobile internet technology has made a great progress. The diversity of devices (PC, mobile, intelligent phones, Personal Digital Assistants) gives rise to the demand for data synchronization; it gradually gets the attention of society.

The concept of data synchronization was derived from storage. Simple synchronization means to perform the same operation, while it defines the object as data. Generally we can treat it as a backup operation among different terminals, the terminal and the server.

The complete data synchronization should be Realtime which means the current operation about two sides is mirror images.

As the rapid development of mobile internet and the popularity of intelligent terminal, data synchronization has been a focus. Concerning about safety and respecting for high-speed transmission have become a strong desire of people. In the traditional data synchronization data should be transmitted to the server firstly, then the server transmits the data to the other clients, which means that the same data need transmit twice; not only it wastes the bandwidths but also reduces the Real-time performance. So with basic control and transmission protocol in mobile internet, we can find a new effective solution to improve the reliability of data synchronization by separating them.

The data synchronization system proposed in this paper diffs from the traditional data synchronization based on client/server. We can consider the new system as a client-to-client system, which transmits the basic request instructions between the clients and the server, and it transmits data directly between clients and clients. The whole system mainly contains two parts: Synchronization Control Server and the Client. The Synchronization Server supports the basic transmission and control protocol, it also should process the instructions which come from clients correctly; it is the hub of the entire system. Through the use of mobile internet, the always online server interacts with the client Real-time and keeps the clients always online when they are transmitting data. Another important component of the system is the Client which sends and receives the data from other clients. The Client mainly includes Login, Authenticating and Data Synchronization. Users can synchronize large amounts of data more safe, reliable and fast with the use of the Client.

The rest of this paper is organized as follows: we describe related work in section II. Section III presents the architecture of the data synchronization system. In section IV, we describe the design principles of the system. Then we display the detail design of data synchronization system in section V. In the second section from the bottom we describe the specific procedure of the system. Finally we conclude in section VII.

\section{Related Work}

Data synchronization is the process of establishing consistency among data from a source to target data storage and vice versa and the continuous harmonization of the data over time. It is fundamental to a wide variety of applications, including file synchronization and mobile device synchronization for PDAs (Personal Digital As- 
sistants).[1] At present, China, USA (United States of America), EU (European Union) and other countries have realized the huge economic benefits of data synchronization, and they are all spending lots of effort to promote and apply it.

Personal digital assistants and other mobile computing devices rely on synchronization protocols in order to maintain data consistency. These protocols operate in environments where network resources such as bandwidth, memory and processing power are limited. [2]

The Session Initiation Protocol (SIP) is an IETF (Internet Engineering Task Force) defined signaling protocol widely used for controlling communication sessions such as voice and video calls over Internet Protocol (IP), the protocol can be used for creating, modifying and terminating two-party (unicast) or multiparty (multicast) sessions. The SIP protocol is an Application Layer protocol designed to be independent of the underlying Transport Layer; it can run on Transmission Control Protocol (TCP), User Datagram Protocol (UDP), or Stream Control Transmission Protocol (SCTP).[3]

SyncML (Synchronization Markup Language) is a specification for a common data synchronization framework for synchronizing data on networked devices. SyncML is designed for use between mobile devices that are intermittently connected to the network and network services that are continuously available on the network; we have designed and developed the data synchronization system based on the SyncML protocol. [4] SyncML is most commonly thought of as a method to synchronize contact and calendar information (personal information manager) between some type of handheld device and a computer (personal, or network-based service), such as between a mobile phone and a personal computer. The new version of the specification includes support for push email, providing a standard protocol alternative to proprietary solutions like BlackBerry.

Rsync is a software application network protocol for Unix-like and Windows systems that synchronizes files and directories from one location to another while minimizing data transfer using delta encoding when appropriate. An important feature of rsync not found in most similar programs/protocols is that the mirroring takes place with only one transmission in each direction. Rsync can copy or display directory contents and copy files, optionally using compression and recursion. The open-source rsync utility reduces the time and bandwidth required to update data across a network. Rsync uses an interactive protocol that detects changes and sends only the changed data $[5,6]$.

Traditional data synchronization system shown in Figure 1 synchronizes data with SyncML which is based on C/S. It contains Central Database Server, Synchronization Server, Web Server and the Client. Among it, the
Central Database Server uses LADP (Lightweight Directory Access Protocol) which is a special kind of database system, specially optimizes for reading, browsing and searching operation, widely used in basic management of critical information, such as user information, network resource information. Data on the Central Database Server can be modified in two ways:

1) The fixed clients modify the records in the central database through a web page by the Web Server.

2) The mobile clients access the central database and synchronize with it through the Synchronization Server.

The main procedures of synchronization in Figure 1 can be elaborated as follows:

First of all, if the mobile client wants to synchronize data with the fixed client, the former will send the synchronization request to the synchronization server, after the server processes the request, the connection between the mobile client and the sever is established, if the other fixed client is online at the same time, the server would also send the synchronization request to it; if the fixed client is offline, then the server will store the request until it is online next time.

Secondly, after the connection is established, the mobile client synchronizes data with the server according to corresponding synchronization protocol and algorithm, meanwhile; the data will be stored into the central database server.

Finally, the server will send the changed data to the fixed client, if it does not change any data, some data will be added or deleted according to the synchronization request; if the fixed client changed some data when the mobile client is synchronizing data with the server, then an conflict will appear on the server, the server then produces the same conflict data to prevent the loss of data.

\section{Architecture of New Data Synchronization System}

The new data synchronization system is developed based on the traditional system by using control protocol SIP and transmission protocol SyncML or rsync. The new data synchronization system contains two parts: Server and Client. The components are shown in Figure 2.

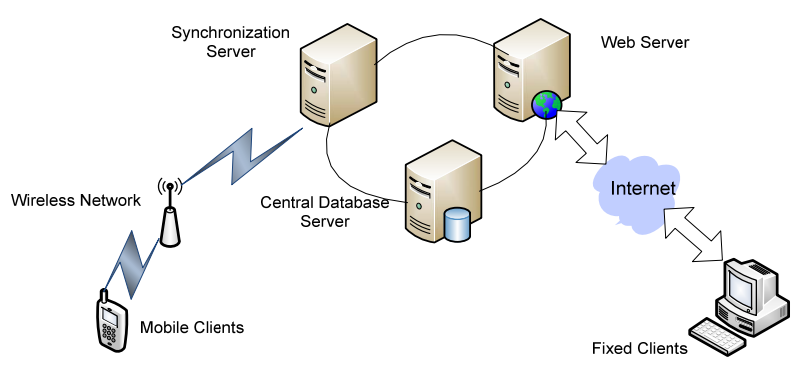

Figure 1. The traditional data synchronization system. 


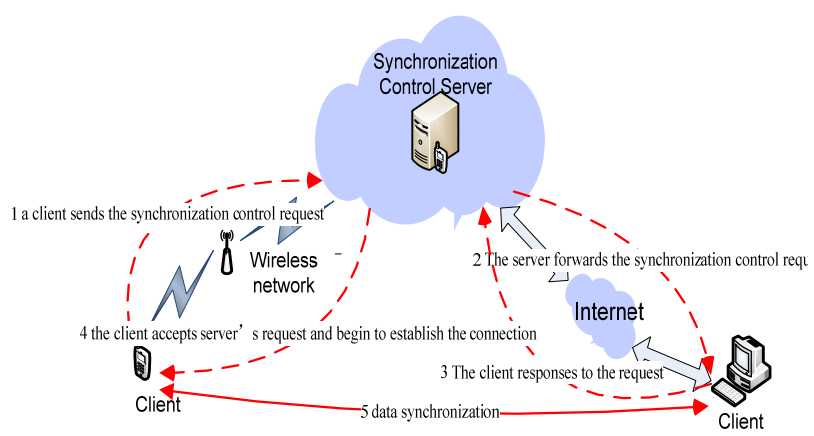

Figure 2. The new data synchronization system.

\subsection{Server}

The synchronization control server is mainly used to processes and forwards the requests from the client. First of all, all the clients must send the register information (IP, Port and so on) to the server before they synchronize data, it will store the key information from the request, after storing the register information, the client will send the control information (IP, Device Name, Data Type and so on) to the server again, the server then processes the client's data to the other client, finally, if both the clients receives control information from the server, then the client will begin to synchronize data.

\subsection{Client}

The component of the client can be the mobile client or the fixed client, which synchronizes the data from the client to the other client by synchronization protocol. The client can choose the other client by searching all the online clients. When the client wants to synchronize data, it just needs send control request to the server and waits for responses. After receiving responses, the client can synchronize all the data what the clients want.

\section{Design Principles of Data Sychronization System}

This paper provides a novel data synchronization methods and system, we adopt to synchronize data by separating control and transmission, detail is described as follows:

First of all the synchronization control server receives the clients' registration request and store the key user information from the request, then the synchronization control server forwards data synchronization session information between the synchronization clients according to its own storage, on the basis of the session information, direct data transmission is carried out between the synchronization clients.

Depending on the context of the synchronization session that is established between the clients, the synchronization protocol (SyncML or rsync) can be adopted to complete each type of data synchronization. The synchronization session information can be described with XML (Extensible Markup Language). [7] A concrete example can be illustrated as follows:

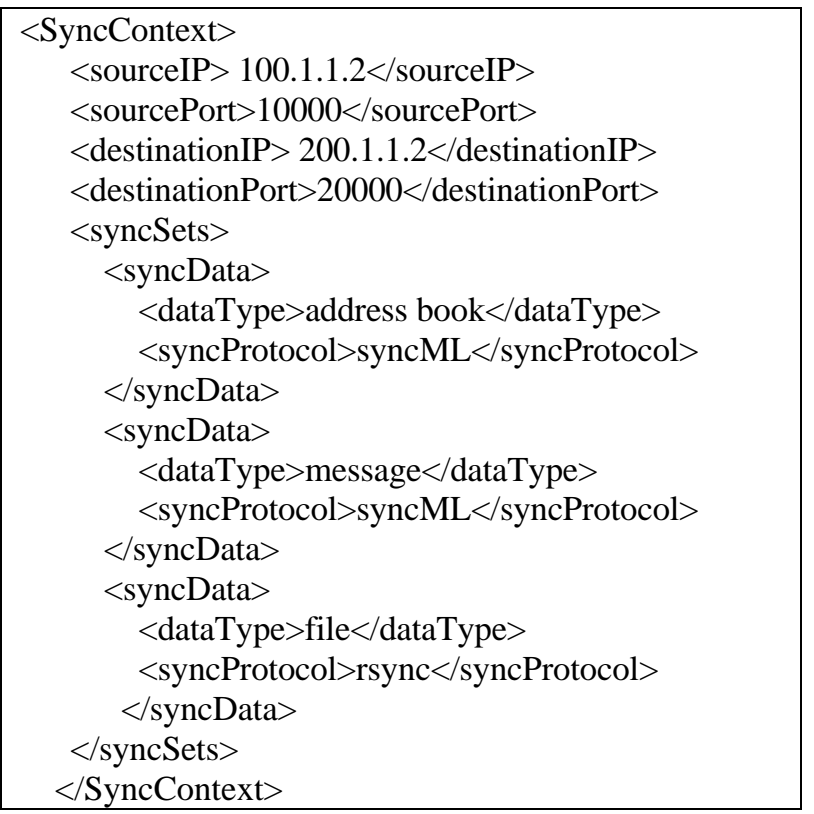

As is seen, users do not need to save their own data on the server in this way, if the client wants to synchronize data with the other client, the client only need to send control request command to the server, when the server accepts it, the server will process and forward it to the other client, if the control flow is established, the client can transmit data bypass the server side, thereby the pressure on the server side is significantly reduced.

In addition, because the process of data synchronization separates the control and the transmission, real time is ensured and the efficiency is improved. Moreover, since the data is stored in the client, it also provides greater robustness and security for the data synchronization

\section{Design Architecure of Data Synchronization System}

The whole architecture of the data synchronization system is shown in Figure 3. The detail framework instruction of the Client is illustrated in part A and the mainly functions in Server is shown in part B.

\subsection{Framework of the Client}

The whole client is divided into three layers: Application, Bearing and Transport. We will make a brief introduction of these three layers in the following.

1) Application Layer

The Application Layer is mainly to interact with users. It helps user to choose what they want (SMS, vcard, vca- 
lendar, picture, $\mathrm{mp} 3$ and so on) to synchronize the other client. This layer includes SIP, which mainly controls the register information and data synchronization session to keep the clients' information, and the Always Online Protocol that keeps the client online.

2) Bearing Layer

The Bearing Layer is responsible for business logic processing in the system; it connects the Application Layer and the Transport Layer like a bridge. It mainly includes the HTTP.

3) Transport Layer

The Transport Layer is mainly to establish TCP/IP connection with other client, used to send data and receive data. The transport layer is responsible for delivering data to the appropriate application process on the computers or phones. This involves statistical multiplexing of data from different application processes, i.e. forming data packets, and adding source and destination port numbers in the header of each transport-layer data packet.

\subsection{Server Functions}

The server of data synchronization system contains two modules: Business Module and Management Module.

1) Business Module

This module consists of two parts: SIP Control Server and AOL Server, the former mainly provides the registration, login and control function. The latter mainly keeps the client online while synchronizing data.

During the registration process, if the user is not registered, the user can register to the server by inputting username and password into the client, at the same time, the IP and the device name is recorded in the server.

During the process of login, if the registration above is successful, then the user can sign in; otherwise an error message will be popped up on the screen. Moreover, this

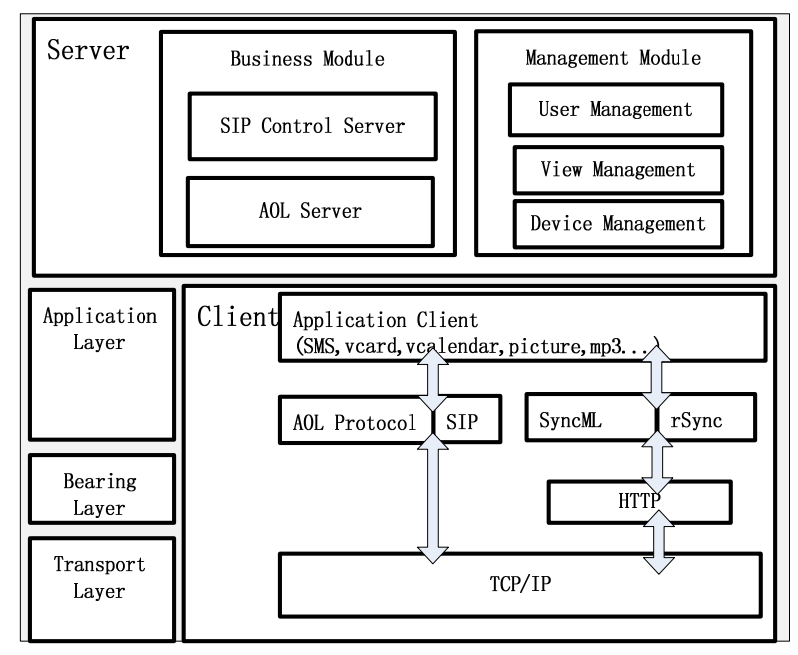

Figure 3. The architecture of data synchronization system. module also offers the setting function which enables users to set the IP address of server and save one's username and password.

During the control process, when the user chooses to synchronize the data, the control module will work, then an dialogue will be shown to the user, in this dialogue, other registered online clients will be displayed to the user, user can choose which clients they want to build the connection.

2) Management Function

The module includes user management, view management and device management.

User management is used to verify the user authority, it also offers the basic function which adds or deletes one user by the administrator.

View management is mainly to interact with an administrator to interact with administrator who can also monitor the clients' synchronization progress conveniently.

Device Management is to add, modify or delete one device by the administrator; the administrator can also examine detailed information about each device.

\section{Specific Procedure}

As is seen the detail design principles and architecture above, we will describe the detail procedure (shown in Figure 4) in this section. The flow chart mainly contains four stages, we will introduce them respectively.

1) Synchronization Server Register / Always Online

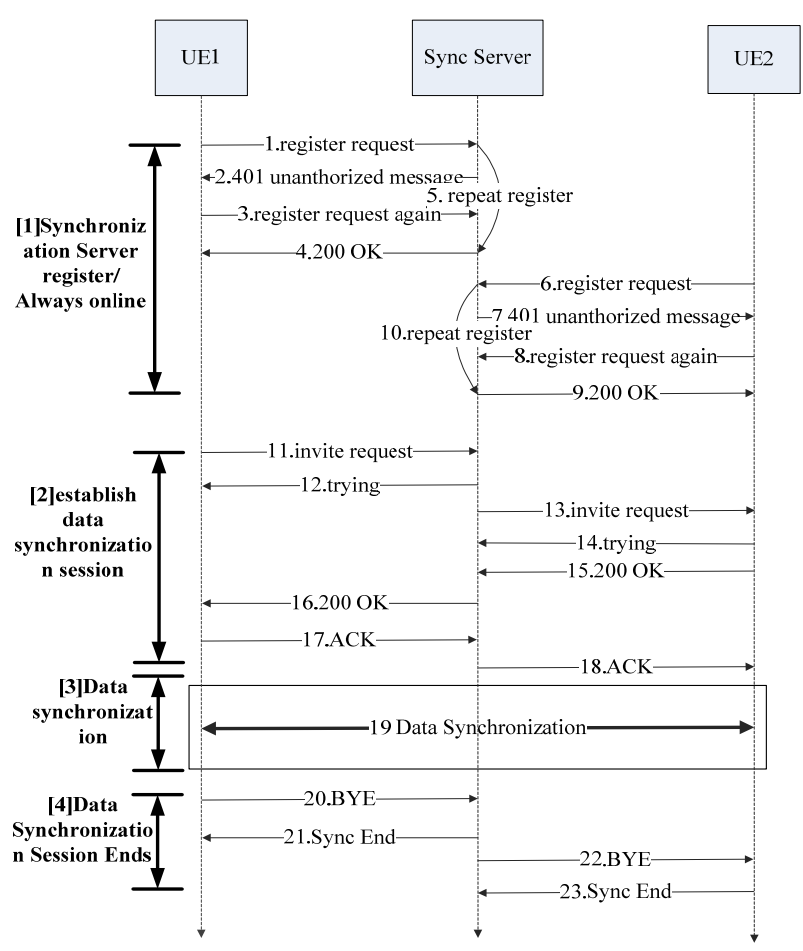

Figure 4. The detail procedure of data synchronization. 
In Figure 4, step1- step10 corresponds to this stage; the user device sends periodic registration to the synchronization control server to keep the client online that means to maintain the user device's IP address, port and other information stored in the synchronization control server. The user device can also send the registration information which contains the changed message to the server if changing is detected. The always online module, receiving the synchronization client's message, handles them and keeps the client online.

Step 1: UE1 sends unauthorized register request to the synchronization server, so as to the Server can get the register information. Described in the general registration information includes the user device's IP address, port number.

Here the message can be carried with SIP.

Step 2: Synchronization control server authenticates it through the back-end, if the user's IP address is not in the database, then the Server will send the unauthenticated 401 message to UE1, secure authentication token is required.

Step 3: According to the token, UE1 marks it and encrypts the password, it will send authorized register request to the Server.

Step 4: The Server accepts this register request, decrypts it and stores it into local database, then it will send 200 OK message in response.

Step 5: After successful registration, the client will repeat sending the register request to the Server to keep it online, the interval generally is 60 seconds.

Step 6-10: UE2 will send the same register request to UE1, after successful registration it will repeat sending register request to the Server to keep online.

2) Establish data synchronization session

Through the synchronization control server, the user device interacts data synchronization session information with other user device, so the data synchronization session is established between the user devices. This stage corresponds to step11-step18 in Figure 4.

Step 11: After successful register, UE1 sends the invite request message which contains basic session information such as Type and Protocol and so on.

Step 12: The synchronization server sends trying message which means the invite message is being processed.

Step 13: UE1 will be authenticated, if it is certified, the Server will check via header field of the invite message whether it contains the IP address of UE1, if it carries, then the Server will insert its own IP address to the via header field, finally, the Server will put forward the invite message to UE2.

Step 14: UE2 accepts the invite message and sends trying message which means it is processing it.

Step 15: If UE2 has got the message, it will match the data type and the protocol, and packages its own message
(IP address, port) into the $200 \mathrm{OK}$ messages.

Step 16: The Server gets the 200 OK messages and forwards it to UE1.

Step 17: After UE1 receives the 200 OK messages, it will send acknowledge message to ensure secure and reliable connection.

Step18: UE2 receives the acknowledge message to confirm it, so the SIP control connection is established, and it is ready for synchronizing data.

3) Data synchronization

This stage corresponds to step 19 in Figure 4, according to data synchronization session mentioned above, directly data transmission is conducted between the two devices, which the synchronization protocol can be SyncML or rsync on the basis of different data types.

Step 19: According to data synchronization session information UE1 will choose consulted synchronization protocol with UE2 against different data types, then begins to synchronize data.

4) Data synchronization ends

In the last four steps (step 20-step23) in Figure 4, when we need to end the data synchronization session, the user device can end the session with the other device by the synchronization control server.

Step 20: UE1 sends bye message to the Synchronization Server to request to end the synchronization this time.

Step 21: The Synchronization receives the bye message and response it to end this connection.

Step 22: The Synchronization Server sends bye message to UE2 to end this connection and prepares for recycling the session information; after receiving the 200 OK messages from UE2, the Server will delete related information about synchronization session information, so it realizes to recycle them above.

Step 23: UE2 ends the synchronization transmission between UE1 and UE2, finally, it will send 200 OK messages to ensure successful conclusion of the synchronous data.

\section{Conclusions}

The development of mobile internet and intelligent terminal bring us many new ways to improve the efficiency and the speed of data synchronization. We can use these technologies to set up a comprehensive and effective synchronization system to synchronize data timely. This system should be much helpful for those clients to have a high speed to synchronize data and to improve efficiency.

\section{Acknowledgements}

The work presented in this paper was supported by the National Grand Fundamental Research 973 Program of 
China (Grant No.2011CB302506); National Natural Science Foundation of China (Grant No.61001118, 61132001, 61171102, 61003067); Project of New Generation Broadband Wireless Network (Grant No.2010ZX03004-001); Program for New Century Excellent Talents in University (Grant No. NCET-11-0592).

\section{REFERENCES}

[1] S. Agarwal, D. Starobinski and A. Trachtenberg, "On the Scalability of Data Synchronization Protocols for PDAs and Mobile Devices,” 2007.

[2] S. Agarwal, D. Starobinski and A. Trachtenberg, "On the Scalability of Data Synchronization Protocols for PDAs and Mobile Devices,” IEEE Network, 2002. doi:10.1109/MNET.2002.1020232

[3] Johnston and B. Alan, "SIP: Understanding the Session Initiation Protocol,” Second Edition.2004.

[4] M. L. Syno, Initiative and M. L. Sync, "Representation Protocol Version 1.1.1,” Oct 29, 2002.

[5] A. Tridgell, "Efficient Algorithms for Sorting and Synchronization," PhD thesis, Australian National University, 1999.

[6] A. Tridgell and P. Mackeras, "The Rsync Algorithm," Technical Report Available at http://samba.anu.edu.au-/rsync/tech report/techreport.html, Australian National University, 1998.

[7] T. Eric, “Learning XML,” Second Edition, 2009. 